\title{
CHARACTERIZATIONS OF LINEAR WEINGARTEN SPACELIKE HYPERSURFACES IN EINSTEIN SPACETIMES
}

\author{
HENRIQUE F. DE LIMA and JOSEÍLSON R. DE LIMA \\ Departamento de Matemática e Estatística, Universidade Federal de Campina Grande, \\ 58429-970 Campina Grande, Paraíba, Brazil \\ e-mails: henrique@dme.ufcg.edu.br, joseilson@dme.ufcg.edu.br
}

(Received 19 March 2012; revised 25 July 2012; accepted 30 August 2012; first published online 25 February 2013)

\begin{abstract}
Our purpose is to study the geometry of linear Weingarten spacelike hypersurfaces immersed in a locally symmetric Einstein spacetime, whose sectional curvature is supposed to obey some standard restrictions. In this setting, by using as main analytical tool a generalized maximum principle for complete non-compact Riemannian manifolds, we establish sufficient conditions to guarantee that such a hypersurface must be either totally umbilical or an isoparametric hypersurface with two distinct principal curvatures, one of which is simple. Applications to the de Sitter space are given.
\end{abstract}

2000 Mathematics Subject Classification. Primary 53C42, Secondary 53A10, 53B30 and 53C50.

1. Introduction. During the last years, the study of spacelike hypersurfaces immersed in a Lorentzian space (that is, a semi-Riemannian manifold of index 1) has been of substantial interest from both physical and mathematical points of view. We recall that a hypersurface $M^{n}$ immersed in a Lorentz space $L_{1}^{n+1}$ is said to be spacelike if the metric on $M^{n}$ induced from that of the ambient space $L_{1}^{n+1}$ is positive definite. From the physical point of view, that interest became clear when Lichnerowicz [18] showed that the Cauchy problem of the Einstein equation with initial conditions on a maximal spacelike hypersurface (that is, with zero mean curvature) has a particularly nice form, reducing to a linear differential system of first order and to a non-linear second-order elliptic differential equation.

From a mathematical point of view, spacelike hypersurfaces are also interesting because of their Bernstein-type properties. As for the case of the de Sitter space $\mathbb{S}_{1}^{n+1}$, which is the standard simply connected Lorentz space form of positive constant sectional curvature 1, Goddard [14] conjectured that every complete spacelike hypersurface with constant mean curvature $H$ in $\mathbb{S}_{1}^{n+1}$ should be totally umbilical. Although the conjecture turned out to be false in its original statement, it motivated a great deal of work of several authors trying to find a positive answer to the conjecture under appropriate additional hypotheses. For instance, in [2] Akutagawa showed that Goddard's conjecture is true when $0 \leq H^{2} \leq 1$ in the case $n=2$, and when $0 \leq H^{2}<4(n-1) / n^{2}$ in the case $n \geq 3$. Later on, Montiel [20] solved Goddard's problem in the compact case, proving that the only closed spacelike hypersurfaces in $\mathbb{S}_{1}^{n+1}$ with constant mean curvature are the totally umbilical hypersurfaces.

Another Goddard-like problem is to characterize spacelike hypersurfaces immersed in a Lorentz space with constant scalar curvature. An interesting result 
due to Cheng and Ishikawa [11] states that the totally umbilical round spheres are the only compact spacelike hypersurfaces in $\mathbb{S}_{1}^{n+1}$ with constant normalized scalar curvature $R<1$. Many other authors, such as Brasil et al. [5], Camargo et al. [7], Caminha [8], Hu et al. [15] and $\mathrm{Li}[\mathbf{1 6}]$ have also worked on related problems.

Proceeding, it is natural to study the geometry of spacelike hypersurfaces immersed in more general Lorentz spaces since they have important meaning in the relativity theory and are of substantial interest from the geometric and cosmological points of view. It is exactly the case of an Einstein spacetime, that is, a Lorentz space whose metric and Ricci tensors are homotetic. On the other hand, for constants $c_{1}$ and $c_{2}$, Choi et al. [13] and Suh et al. [24] have introduced the class of Lorentz spaces $L_{1}^{n+1}$ which satisfy the following two conditions (here $K$ denotes the sectional curvature of $\left.L_{1}^{n+1}\right)$ :

$$
K(u, v)=-\frac{c_{1}}{n}
$$

for any spacelike vector $u$ and timelike vector $v$; and

$$
K(u, v) \geq c_{2}
$$

for any spacelike vectors $u$ and $v$.

We observe that Lorentz space forms $L_{1}^{n+1}(c)$ of constant sectional curvature $c$ satisfy conditions (1.1) and (1.2) for $-\frac{c_{1}}{n}=c_{2}=c$. Moreover, there are several examples of Lorentz spaces which are not Lorentz space forms and satisfy (1.1) and (1.2). For instance, semi-Riemannian product manifolds $\mathbb{W}_{1}^{k}\left(-c_{1} / n\right) \times N^{n+1-k}\left(c_{2}\right)$, where $c_{1}>0$, and $\mathbb{R}_{1}^{k} \times \mathbb{S}^{n+1-k}$. In particular, $\mathbb{R}_{1}^{1} \times \mathbb{S}^{n}$ is the so-called Einstein Static Universe. Also, the so-called Robertson-Walker Spacetime $N(c, f)=I \times_{f} N^{3}(c)$ is another general example of Lorentz space, where $I$ denotes an open interval of $\mathbb{R}_{1}^{1}, f$ is a positive smooth function defined on the interval $I$ and $N^{3}(c)$ is a three-dimensional Riemannian manifold of constant curvature $c . N(c, f)$ also satisfies conditions (1.1) and (1.2) for an appropriate choice of function $f$ (for more details, see [13, 24].

Our purpose is to study the geometry of linear Weingarten spacelike hypersurfaces (that is, spacelike hypersurfaces whose mean curvature $H$ and normalized scalar curvature $R$ satisfy $R=a H+b$, for some $a, b \in \mathbb{R}$ ) immersed in a locally symmetric Einstein spacetime $\mathcal{E}_{1}^{n+1}$ satisfying conditions (1.1) and (1.2). We recall that a Lorentz space is said to be locally symmetric when all the covariant derivative components $\bar{R}_{A B C D ; E}$ of its curvature tensor vanish identically.

In this setting, denoting by $\bar{R}_{A B}$ the components of the Ricci tensor of $L_{1}^{n+1}$ satisfying condition (1.1), the scalar curvature $\bar{R}$ of $L_{1}^{n+1}$ is given by

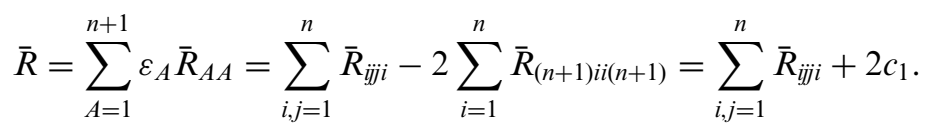

Moreover, it is well known that the scalar curvature of a locally symmetric Lorentz space is constant. Consequently, $\sum_{i, j} \bar{R}_{i j j i}$ is a constant naturally attached to a locally symmetric Lorentz space satisfying condition (1.1).

Now we are in position to present our results.

THEOREM 1.1. Let $\mathcal{E}_{1}^{n+1}$ be a locally symmetric Einstein spacetime satisfying conditions (1.1) and (1.2), with $c=\frac{c_{1}}{n}+2 c_{2}>0$. Let $M^{n}$ be a complete non-compact 
linear Weingarten spacelike hypersurface immersed in $\mathcal{E}_{1}^{n+1}$ such that $R=a H+b$ with $(n-1)^{2} a^{2}+4 \sum_{i, j} \bar{R}_{i j i}-4 n(n-1) b>0$. If $|\nabla H|$ is a Lebesgue integrable on $M^{n}$ and $S \leq 2 \sqrt{n-1} c$, then $M^{n}$ is either totally umbilical or an isoparametric hypersurface with two distinct principal curvatures one of which is simple.

Here $S$ stands for the square length of the second fundamental form of the spacelike hypersurface $M^{n}$. Related to the compact case, we get the following.

THEOREM 1.2. Let $\mathcal{E}_{1}^{n+1}$ be a locally symmetric Einstein spacetime satisfying conditions (1.1) and (1.2), with $c=\frac{c_{1}}{n}+2 c_{2}>0$. Let $M^{n}$ be a compact linear Weingarten spacelike hypersurface immersed in $\mathcal{E}_{1}^{n+1}$ such that $R=a H+b$ with $(n-1)^{2} a^{2}+$ $4 \sum_{i, j} \bar{R}_{\ddot{i j i} i}-4 n(n-1) b \geq 0$. If $S<2 \sqrt{n-1} c$, then $M^{n}$ is totally umbilical.

In Section 3 we present the proofs of Theorems 1.1 and 1.2, as well as their applications when the ambient space is the de Sitter space $\mathbb{S}_{1}^{n+1}$ (cf. Corollaries 3.4 and 3.6). Our approach is based on the use of the well-known Cheng-Yau [12] square operator (cf. Section 2) jointly with a suitable extension of the maximum principle at the infinity of Yau [25] due to Caminha in [9] (cf. Lemma 3.3).

We close our Introduction observing that the previous theorems can be regarded as the extensions of characterization results of the current literature concerning spacelike hypersurfaces with either constant mean curvature or constant scalar curvature in locally symmetric Lorentz spaces. In this sense we refer the readers to the works of Ok Baek et al. [22], Liu and Sun [19] and Zhang and $\mathrm{Wu}$ [26]. Moreover, we point out that Li et al. [17] have obtained rigidity theorems related to linear Weingarten hypersurfaces immersed in the unit Euclidean sphere $\mathbb{S}^{n+1}$.

2. Preliminaries. From now on, we will consider complete spacelike hypersurfaces $M^{n}$ immersed in a Lorentz space $L_{1}^{n+1}$. We choose a local field of semiRiemannian orthonormal frame $\left\{e_{A}\right\}_{1 \leq A \leq n+1}$ in $L_{1}^{n+1}$, with dual coframe $\left\{\omega_{A}\right\}_{1 \leq A \leq n+1}$ such that at each point of $M^{n} e_{1}, \ldots, e_{n}$ are tangent to $M^{n}$ and $e_{n+1}$ is normal to $M^{n}$. We will use the following convention for the indices:

$$
1 \leq A, B, C, \ldots \leq n+1,1 \leq i, j, k, \ldots \leq n .
$$

In this setting, denoting by $\left\{\omega_{A B}\right\}$ the connection forms of $L_{1}^{n+1}$, we have the structure equations of $L_{1}^{n+1}$ given by

$$
\begin{gathered}
d \omega_{A}=-\sum_{B} \varepsilon_{B} \omega_{A B} \wedge \omega_{B}, \quad \omega_{A B}+\omega_{B A}=0, \quad \varepsilon_{i}=1, \varepsilon_{n+1}=-1, \\
d \omega_{A B}=-\sum_{C} \varepsilon_{C} \omega_{A C} \wedge \omega_{C B}-\frac{1}{2} \sum_{C, D} \varepsilon_{C} \varepsilon_{D} \bar{R}_{A B C D} \omega_{C} \wedge \omega_{D} .
\end{gathered}
$$

Here $\bar{R}_{A B C D}, \bar{R}_{C D}$ and $\bar{R}$ denote respectively the Riemannian curvature tensor, the Ricci tensor and the scalar curvature of the Lorentz space $L_{1}^{n+1}$. In this setting, we have

$$
\bar{R}_{C D}=\sum_{B} \varepsilon_{B} \bar{R}_{B C D B}, \quad \bar{R}=\sum_{A} \varepsilon_{A} \bar{R}_{A A}
$$


Moreover, the components $\bar{R}_{A B C D ; E}$ of the covariant derivative of the Riemannian curvature tensor of $L_{1}^{n+1}$ are defined by

$$
\begin{aligned}
\sum_{E} \varepsilon_{E} \bar{R}_{A B C D ; E} \omega_{E}= & d \bar{R}_{A B C D}-\sum_{E} \varepsilon_{E}\left(\bar{R}_{E B C D} \omega_{E A}\right. \\
& \left.+\bar{R}_{A E C D} \omega_{E B}+\bar{R}_{A B E D} \omega_{E C}+\bar{R}_{A B C E} \omega_{E D}\right)
\end{aligned}
$$

Next, we restrict all the tensors to the spacelike hypersurface $M^{n}$ in $L_{1}^{n+1}$. First of all, $\omega_{n+1}=0$ on $M^{n}$, so $\sum_{i} \omega_{(n+1) i} \wedge \omega_{i}=d \omega_{n+1}=0$. Consequently, by Cartan's Lemma $[\mathbf{1 0}]$, there are $h_{i j}$ such that

$$
\omega_{(n+1) i}=\sum_{j} h_{i j} \omega_{j} \quad \text { and } \quad h_{i j}=h_{j i} .
$$

This gives the second fundamental form of $M^{n}, h=\sum_{i, j} h_{i j} \omega_{i} \omega_{j} e_{n+1}$, and its square length $S=\sum_{i, j} h_{i j}^{2}$. Furthermore, the mean curvature $H$ of $M^{n}$ is defined by $H=$ $\frac{1}{n} \sum_{i} h_{i i}$.

The connection forms $\left\{\omega_{i j}\right\}$ of $M^{n}$ are characterized by the structure equations of $M^{n}$ :

$$
\begin{gathered}
d \omega_{i}=-\sum_{j} \omega_{i j} \wedge \omega_{j}, \quad \omega_{i j}+\omega_{j i}=0, \\
d \omega_{i j}=-\sum_{k} \omega_{i k} \wedge \omega_{k j}-\frac{1}{2} \sum_{k, l} R_{i j k l} \omega_{k} \wedge \omega_{l},
\end{gathered}
$$

where $R_{i j k l}$ are the components of the curvature tensor of $M^{n}$.

Using the structure equations, we obtain the Gauss equation

$$
R_{i j k l}=\bar{R}_{i j k l}-\left(h_{i k} h_{j l}-h_{i l} h_{j k}\right) .
$$

The components $R_{i j}$ of the Ricci tensor and the scalar curvature $R$ of $M^{n}$ are given, respectively, by

$$
R_{i j}=\sum_{k} \bar{R}_{k i j k}-n H h_{i j}+\sum_{k} h_{i k} h_{k j}
$$

and

$$
n(n-1) R=\sum_{j, k} \bar{R}_{k i j k}-n^{2} H^{2}+S .
$$

The first covariant derivatives $h_{i j k}$ of $h_{i j}$ satisfy

$$
\sum_{k} h_{i j k} \omega_{k}=d h_{i j}-\sum_{k} h_{i k} \omega_{k j}-\sum_{k} h_{j k} \omega_{k i} .
$$

Then by exterior differentiation of (2.3), we obtain the Codazzi equation

$$
h_{\ddot{j} k}-h_{i k j}=\bar{R}_{(n+1) \ddot{j} k} \text {. }
$$


Similarly, the second covariant derivatives $h_{i j k l}$ of $h_{i j}$ are given by

$$
\sum_{l} h_{i j k l} \omega_{l}=d h_{i j k}-\sum_{l} h_{l j k} \omega_{l i}-\sum_{l} h_{i l k} \omega_{l j}-\sum_{l} h_{i j l} \omega_{l k}
$$

By exterior differentiation of (2.9), we can get the following Ricci formula

$$
h_{i j k l}-h_{i j l k}=-\sum_{m} h_{i m} R_{m j k l}-\sum_{m} h_{j m} R_{m i k l}
$$

Restricting the covariant derivative $\bar{R}_{A B C D ; E}$ of $\bar{R}_{A B C D}$ on $M^{n}$, then $\bar{R}_{(n+1) i j k ; l}$ is given by

$$
\begin{aligned}
\bar{R}_{(n+1) \dot{j} k ; l}= & \bar{R}_{(n+1) \dot{j} k l}+\bar{R}_{(n+1) i(n+1) k} h_{j l} \\
& +\bar{R}_{(n+1) \ddot{j}(n+1)} h_{k l}+\sum_{m} \bar{R}_{m \ddot{j} k} h_{m l},
\end{aligned}
$$

where $\bar{R}_{(n+1) i j k l}$ denotes the covariant derivative of $\bar{R}_{(n+1) i j k}$ as a tensor on $M^{n}$ so that

$$
\sum_{l} \bar{R}_{(n+1) \dot{j} k l} \omega_{l}=d \bar{R}_{(n+1) \dot{j} k}-\sum_{l} \bar{R}_{(n+1) j k} \omega_{l i}-\sum_{l} \bar{R}_{(n+1) i l k} \omega_{l j}-\sum_{l} \bar{R}_{(n+1) \dot{j} l} \omega_{l k} .
$$

The Laplacian $\Delta h_{i j}$ of $h_{i j}$ is defined by $\Delta h_{i j}=\sum_{k} h_{i j k k}$. From (2.10), (2.12) and (2.13), after a straightforward computation, we obtain

$$
\begin{aligned}
\Delta h_{i j}= & (n H)_{i j}-n H \sum_{l} h_{i l} h_{l j}+S h_{i j} \\
& +\sum_{k}\left(\bar{R}_{(n+1) \ddot{j} k ; k}+\bar{R}_{(n+1) k i k ; j}\right) \\
& -\sum_{k}\left(h_{k k} \bar{R}_{(n+1) \ddot{j}(n+1)}+h_{i j} \bar{R}_{(n+1) k(n+1) k}\right) \\
& -\sum_{k, l}\left(2 h_{k l} \bar{R}_{l i j k}+h_{j l} \bar{R}_{l k i k}+h_{i l} \bar{R}_{l k j k}\right) .
\end{aligned}
$$

Since $\Delta S=2\left(\sum_{i, j, k} h_{i j k}^{2}+\sum_{i, j} h_{i j} \Delta h_{i j}\right)$, from (2.14) we get

$$
\begin{aligned}
\frac{1}{2} \Delta S= & S^{2}+\sum_{i, j, k} h_{i j k}^{2}+\sum_{i, j}(n H)_{i j} h_{i j} \\
& +\sum_{i, j, k}\left(\bar{R}_{(n+1) i j k ; k}+\bar{R}_{(n+1) k i k ; j}\right) h_{i j} \\
& -\left(\sum_{i, j} n H h_{i j} \bar{R}_{(n+1) \ddot{j}(n+1)}+S \sum_{k} \bar{R}_{(n+1) k(n+1) k}\right) \\
& -2 \sum_{i, j, k, l}\left(h_{k l} h_{i j} \bar{R}_{l i j k}+h_{i l} h_{i j} \bar{R}_{l k j k}\right)-n H \sum_{i, j, l} h_{i l} h_{l j} h_{i j} .
\end{aligned}
$$


Now let $\phi=\sum_{i, j} \phi_{i j} \omega_{i} \otimes \omega_{j}$ be a symmetric tensor on $M^{n}$ defined by

$$
\phi_{i j}=n H \delta_{i j}-h_{i j} .
$$

Following Cheng-Yau [12], we introduce an operator $\square$ associated to $\phi$ acting on any smooth function $f$ by

$$
\square f=\sum_{i, j} \phi_{i j} f_{i j}=\sum_{i, j}\left(n H \delta_{i j}-h_{i j}\right) f_{i j}
$$

Setting $f=n H$ in (2.16) and taking a (local) orthonormal frame $\left\{e_{1}, \ldots, e_{n}\right\}$ on $M^{n}$ such that $h_{i j}=\lambda_{i} \delta_{i j}$, from equation (2.8) we obtain the following:

$$
\begin{aligned}
\square(n H)= & \frac{1}{2} \Delta(n H)^{2}-\sum_{i}(n H)_{i}^{2}-\sum_{i} \lambda_{i}(n H)_{i i} \\
= & \frac{1}{2} \Delta S-n^{2}|\nabla H|^{2}-\sum_{i} \lambda_{i}(n H)_{i i} \\
& +\frac{1}{2} \Delta\left(\sum_{i, j} \bar{R}_{\dddot{j} j i}-n(n-1) R\right) .
\end{aligned}
$$

3. Proofs of Theorems 1.1 and 1.2 and their applications in $\mathbb{S}_{1}^{n+1}$. In order to prove our results, we will need some auxiliary lemmas. The first one is a classic algebraic lemma due to Okumura in [23], and completed with the equality case proved in [3] by Alencar and Carmo.

LEMmA 3.1. Let $\mu_{1}, \ldots \mu_{n}$ be real numbers such that $\sum_{i} \mu_{i}=0$ and $\sum_{i} \mu_{i}^{2}=\beta^{2}$, where $\beta$ is constant and $\beta \geq 0$. Then

$$
-\frac{(n-2)}{\sqrt{n(n-1)}} \beta^{3} \leq \sum_{i} \mu_{i}^{3} \leq \frac{(n-2)}{\sqrt{n(n-1)}} \beta^{3},
$$

and equality holds if, and only if, either at least $(n-1)$ of the numbers $\mu_{i}$ are equal to $\beta / \sqrt{(n-1) n}$ or at least $(n-1)$ of the numbers $\mu_{i}$ are equal to $-\beta / \sqrt{(n-1) n}$.

Now we present our second auxiliary lemma. Following the steps of the proof of Lemma 2.1 in [17], we get the following:

LEMMA 3.2. Let $M^{n}$ be a linear Weingarten spacelike hypersurface immersed in a locally symmetric Lorentz space $L_{1}^{n+1}$ satisfying condition (1.1), such that $R=a H+b$. Suppose that

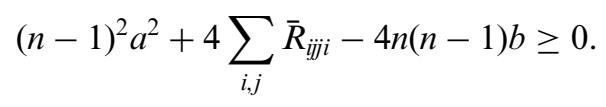


Then,

$$
\sum_{i, j, k} h_{i j k}^{2} \geq n^{2}|\nabla H|^{2}
$$

Moreover, if the inequality (3.2) is strict and the equality holds in (3.3) on $M^{n}$, then $H$ is constant on $M^{n}$.

Proof. Since we are supposing that $R=a H+b$ and the term is $\sum_{i, j} \bar{R}_{i j j i}$ constant, from equation (2.8) we get

$$
2 \sum_{i, j} h_{i j} h_{i j k}=\left(2 n^{2} H+n(n-1) a\right)(H)_{k} .
$$

Thus,

$$
4 \sum_{k}\left(\sum_{i, j} h_{i j} h_{i j k}\right)^{2}=\left(2 n^{2} H+n(n-1) a\right)^{2}|\nabla H|^{2} .
$$

Consequently, using the Cauchy-Schwartz inequality, we obtain

$$
\begin{aligned}
4 S \sum_{i, j, k} h_{i j k}^{2} & =4\left(\sum_{i, j} h_{i j}^{2}\right)\left(\sum_{i, j, k} h_{i j k}^{2}\right) \\
& \geq 4 \sum_{k}\left(\sum_{i, j} h_{i j} h_{i j k}\right)^{2} \\
& =\left(2 n^{2} H+n(n-1) a\right)^{2}|\nabla H|^{2} .
\end{aligned}
$$

On the other hand, since $R=a H+b$, using again equation (2.8) we easily verify that

$$
\begin{aligned}
\left(2 n^{2} H+n(n-1) a\right)^{2}= & 4 n^{2} \sum_{i, j} \bar{R}_{i j i}-4 n^{3}(n-1) b \\
& +n^{2}(n-1)^{2} a^{2}+4 n^{2} S .
\end{aligned}
$$

Consequently, from (3.2), (3.4) and (3.5), we get

$$
S \sum_{i, j, k} h_{i j k}^{2} \geq n^{2} S|\nabla H|^{2} .
$$

Therefore, we obtain either $S=0$ and $\sum_{i, j, k} h_{i j k}^{2}=n^{2}|\nabla H|^{2}$ or $\sum_{i, j, k} h_{i j k}^{2} \geq n^{2}|\nabla H|^{2}$. Moreover, if the inequality (3.2) is strict, from (3.5) we get

$$
\left(2 n^{2} H+n(n-1) a\right)^{2}>4 n^{2} S .
$$

Consequently, if $\sum_{i, j, k} h_{i j k}^{2}=n^{2}|\nabla H|^{2}$ holds on $M^{n}$, from (3.4) we conclude that $\nabla H=$ 0 on $M^{n}$, and hence $H$ is constant on $M^{n}$.

Yau in [25] established the following version of Stokes' Theorem on an $n$ dimensional, complete non-compact Riemannian manifold $M^{n}$ : if $\omega \in \Omega^{n-1}(M)$ is 
an $(n-1)$-differential form on $M^{n}$, then there exists a sequence $B_{i}$ of domains on $M^{n}$ such that $B_{i} \subset B_{i+1}, M^{n}=\bigcup_{i \geq 1} B_{i}$ and

$$
\lim _{i \rightarrow+\infty} \int_{B_{i}} d \omega=0 .
$$

Now suppose that $M^{n}$ is oriented by the volume element $d M$. If $\omega=\iota_{X} d M$ is the contraction of $d M$ in the direction of a smooth vector field $X$ on $M^{n}$, then Caminha (cf. [9, Proposition 2.1]) obtained a suitable consequence of Yau's [25] result, which is described below. In what follows, $\mathcal{L}^{1}(M)$ stands for the space of Lebesgue integrable functions on $M^{n}$.

LEMmA 3.3. Let $X$ be a smooth vector field on the n-dimensional complete noncompact-oriented Riemannian manifold $M^{n}$ such that the divergence of $X$ on $M^{n}, \operatorname{div} X$, does not change sign. If $|X| \in \mathcal{L}^{1}(M)$, then $\operatorname{div} X=0$.

Proof of Theorem 1.1.

From (2.16) we have

$$
\square f=\operatorname{trace}\left(P_{1} \circ \nabla^{2} f\right),
$$

where denoting by $I$ the identity in the algebra of smooth vector fields on $M^{n}, P_{1}=$ $n H I-h$ and $\nabla^{2} f$ stands for the self-adjoint linear operator metrically equivalent to the hessian of $f$. Thus, by using the standard notation $\langle$,$\rangle for the (induced) metric of$ $M^{n}$, we get

$$
\square f=\sum_{i}\left\langle P_{1}\left(\nabla_{e_{i}} \nabla f\right), e_{i}\right\rangle
$$

where $\left\{e_{1}, \ldots, e_{n}\right\}$ is a local orthonormal frame on $M^{n}$. Consequently, we have

$$
\begin{aligned}
\operatorname{div}\left(P_{1}(\nabla f)\right) & =\sum_{i}\left\langle\left(\nabla_{e_{i}} P_{1}\right)(\nabla f), e_{i}\right\rangle+\sum_{i}\left\langle P_{1}\left(\nabla_{e_{i}} \nabla f\right), e_{i}\right\rangle \\
& =\left\langle\operatorname{div} P_{1}, \nabla f\right\rangle+\square f,
\end{aligned}
$$

where

$$
\operatorname{div} P_{1}:=\operatorname{trace}\left(\nabla P_{1}\right)=\sum_{i}\left(\nabla_{e_{i}} P_{1}\right)\left(e_{i}\right)
$$

On the other hand, since $\mathcal{E}_{1}^{n+1}$ is an Einstein spacetime, there exists a parameter $\lambda$ such that $\overline{\operatorname{Ric}}=\lambda\langle$,$\rangle , where \overline{\text { Ric }}$ denotes the Ricci tensor of $\mathcal{E}_{1}^{n+1}$. Thus, denoting by $\bar{R}$ the curvature tensor of $\mathcal{E}_{1}^{n+1}$, from Lemma 3.1 of [4] we have

$$
\left\langle\operatorname{div} P_{1}, \nabla f\right\rangle=\sum_{i}\left\langle\bar{R}\left(N, e_{i}\right) e_{i}, \nabla f\right\rangle=-\overline{\operatorname{Ric}}(N, \nabla f)=-\lambda\langle N, \nabla f\rangle=0
$$

where $N$ stands for the Gauss mapping of $M^{n}$. Hence, we conclude from (3.6) that

$$
\square f=\operatorname{div}\left(P_{1}(\nabla f)\right) .
$$


Now we consider the Cheng-Yau's [12] modified operator

$$
L=\square+\frac{n-1}{2} a \Delta .
$$

From (3.7), we have

$$
L(n H)=\operatorname{div}(P(\nabla H))
$$

where $P=n P_{1}+\frac{n(n-1)}{2} a I$. Moreover, since $S$ is supposed to be bounded, we easily verify that the operator $P$ is bounded. Consequently, since we are also assuming that $|\nabla H| \in \mathcal{L}^{1}(M)$, we obtain

$$
|P(\nabla H)| \in \mathcal{L}^{1}(M) .
$$

Now we will obtain a suitable lower estimate for $L(n H)$. For this we observe that the local symmetry of $L_{1}^{n+1}$ implies that

$$
\sum_{i, j, k}\left(\bar{R}_{(n+1) i j k ; k}+\bar{R}_{(n+1) k i k ; j}\right) h_{i j}=0 .
$$

Consequently, if we choose a (local) orthonormal frame $\left\{e_{1}, \ldots, e_{n}\right\}$ on $M^{n}$ such that $h_{i j}=\lambda_{i} \delta_{i j}$, taking into account equations (2.15) and (2.17), and the fact that the term $\sum_{i, j} \bar{R}_{i \dddot{j} i}$ is constant, we get from (3.8)

$$
\begin{aligned}
L(n H)= & \sum_{i, j, k} h_{i j k}^{2}-n^{2}|\nabla H|^{2}+S^{2}-n H \sum_{i} \lambda_{i}^{3} \\
& -2 \sum_{i, k}\left(\lambda_{i} \lambda_{k} \bar{R}_{k i i k}+\lambda_{i}^{2} \bar{R}_{i k i k}\right) \\
& -\left(\sum_{i} n H \lambda_{i} \bar{R}_{(n+1) i i(n+1)}+S \sum_{k} \bar{R}_{(n+1) k(n+1) k}\right) .
\end{aligned}
$$

Thus, from Lemma 3.2, we have

$$
\begin{aligned}
L(n H) \geq & S^{2}-n H \sum_{i} \lambda_{i}^{3}-2 \sum_{i, k}\left(\lambda_{i} \lambda_{k} \bar{R}_{k i i k}+\lambda_{i}^{2} \bar{R}_{i k i k}\right) \\
& -\left(\sum_{i} n H \lambda_{i} \bar{R}_{(n+1) i(n+1)}+S \sum_{k} \bar{R}_{(n+1) k(n+1) k}\right) .
\end{aligned}
$$

Now set $\Phi_{i j}=h_{i j}-H \delta_{i j}$. We will consider the following symmetric tensor,

$$
\Phi=\sum_{i, j} \Phi_{i j} \omega_{i} \otimes \omega_{j} .
$$

Let $|\Phi|^{2}=\sum_{i, j} \Phi_{i j}^{2}$ be the square of the length of $\Phi$. It is easy to check that $\Phi$ is traceless and

$$
|\Phi|^{2}=S-n H^{2}
$$


If we take a (local) frame field $e_{1}, \ldots, e_{n}$ at $p \in M^{n}$ such that

$$
h_{i j}=\lambda_{i} \delta_{i j} \text { and } \Phi_{i j}=\mu_{i} \delta_{i j},
$$

it is straightforward to check that

$$
\sum_{i} \mu_{i}=0, \sum_{i} \mu_{i}^{2}=|\Phi|^{2} \quad \text { and } \quad \sum_{i} \mu_{i}^{3}=\sum_{i} \lambda_{i}^{3}-3 H|\Phi|^{2}-n H^{3} .
$$

Consequently, by applying Lemma 3.1 to the real numbers $\mu_{1}, \ldots, \mu_{n}$, we get

$$
\begin{aligned}
S^{2}-n H \sum_{i} \lambda_{i}^{3}= & \left(|\Phi|^{2}+n H^{2}\right)^{2}-n^{2} H^{4} \\
& -3 n H^{2}|\Phi|^{2}-n H \sum_{i} \mu_{i}^{3} \\
\geq & |\Phi|^{4}-n H^{2}|\Phi|^{2}-\frac{n(n-2)}{\sqrt{n(n-1)}} H|\Phi|^{3} .
\end{aligned}
$$

Using curvature conditions (1.1) and (1.2), we get

$$
-\left(\sum_{i, j} n H \lambda_{i} \bar{R}_{(n+1) i i(n+1)}+S \sum_{k} \bar{R}_{(n+1) k(n+1) k}\right)=c_{1}\left(S-n H^{2}\right)
$$

and

$$
\begin{aligned}
-2 \sum_{i, j, k, l}\left(\lambda_{i} \lambda_{k} \bar{R}_{k i i k}+\lambda_{i}^{2} \bar{R}_{i k i k}\right) & \geq c_{2} \sum_{i, k}\left(\lambda_{i}-\lambda_{k}\right)^{2} \\
& =2 n c_{2}\left(S-n H^{2}\right) .
\end{aligned}
$$

Hence, setting $c=\frac{c_{1}}{n}+2 c_{2}$, from (3.12), (3.13), (3.14) and (3.15) we obtain

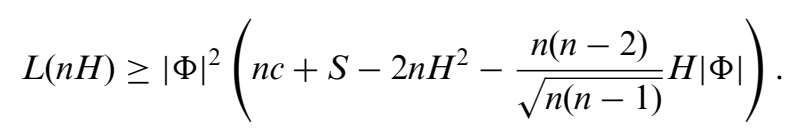

On the other hand, with a straightforward computation we verify that

$$
\begin{aligned}
S-2 n H^{2}= & \frac{1}{2 \sqrt{n-1}}((\sqrt{n-1}+1)|\Phi|-(\sqrt{n-1}-1) \sqrt{n} H)^{2} \\
& +\frac{n(n-2)}{\sqrt{n(n-1)}} H|\Phi|-\frac{n}{2 \sqrt{n-1}} S .
\end{aligned}
$$

Thus, since we are supposing that $S \leq 2 \sqrt{n-1} c$, from (3.16) we get

$$
L(n H) \geq|\Phi|^{2}\left(n c-\frac{n}{2 \sqrt{n-1}} S\right) \geq 0 .
$$

Hence, taking into account (3.9), (3.10) and (3.17), we can apply Lemma 3.3 to conclude that $L(n H)=0$, and from (3.11) we get

$$
\sum_{i, j, k} h_{i j k}^{2}=n^{2}|\nabla H|^{2}
$$


Consequently, since we are assuming that $(n-1)^{2} a^{2}+4 \sum_{i, j} \bar{R}_{i j i}-4 n(n-1) b>0$, it follows from Lemma 3.2 that $H$ is constant. Moreover, from (3.17) we have

$$
|\Phi|^{2}\left(n c-\frac{n}{2 \sqrt{n-1}} S\right)=0
$$

If $S<2 \sqrt{n-1} c$, then $|\Phi|^{2}=0$ and $M^{n}$ is totally umbilical. If $S=2 \sqrt{n-1} c$, since all the inequalities that we have obtained are in fact equalities, we easily verify that

$$
|\Phi|=\frac{(\sqrt{n-1}-1) \sqrt{n}}{\sqrt{n-1}+1} H .
$$

Thus, in the case that $n=2$, from (3.19) we obtain that $|\Phi|^{2}=0$. Hence, $M^{2}$ is totally umbilical.

Finally, when $n \geq 3$, since the equality holds in (3.1) of Lemma 3.1, we conclude that $M^{n}$ is either totally umbilical or an isoparametric hypersurface with two distinct principal curvatures, one of which is simple.

From Theorem 1.1 and according to the classical congruence theorem due to Abe et al. (cf. [1, Theorem 5.1]), we obtain the following result in the de Sitter space $\mathbb{S}_{1}^{n+1}$.

COROLlary 3.4. Let $M^{n}$ be a complete non-compact linear Weingarten spacelike hypersurface immersed in $\mathbb{S}_{1}^{n+1}$ such that $R=a H+b$ with $(n-1) a^{2}+4 n(1-b)>0$. If $S \leq 2 \sqrt{n-1}$ and $|\nabla H| \in \mathcal{L}^{1}(M)$, then $M^{n}$ is either totally umbilical or is isometric to a hyperbolic cylinder $\mathbb{W}^{1}\left(c_{1}\right) \times \mathbb{S}^{n-1}\left(c_{2}\right)$, for $R>0$, or to $\mathbb{W}^{n-1}\left(c_{1}\right) \times \mathbb{S}^{1}\left(c_{2}\right)$, for $R<0$, where $c_{1}<0, c_{2}>0$ and $\frac{1}{c_{1}}+\frac{1}{c_{2}}=1$.

REMARK 3.5. Montiel in [21] characterized the hyperbolic cylinders as the only complete non-compact spacelike hypersurfaces in $\mathbb{S}_{1}^{n+1}$ with constant mean curvature $H=\frac{2 \sqrt{n-1}}{n}$ and having at least two ends. Later on, Brasil et al. [6] obtained a sort of extension of Montiel's result, showing that the hyperbolic cylinders are the only complete spacelike hypersurfaces in $\mathbb{S}_{1}^{n+1}$ with constant mean curvature, non-negative Ricci curvature and having at least two ends. They also characterized all complete spacelike hypersurfaces of constant mean curvature with two distinct principal curvatures as rotation hypersurfaces or generalized hyperbolic cylinders $\dddot{M}^{k}\left(c_{1}\right) \times \mathbb{S}^{n-k}\left(c_{2}\right)$, where $1<k<(n-1), c_{1}<0, c_{2}>0$ and $\frac{1}{c_{1}}+\frac{1}{c_{2}}=1$.

Proof of Theorem 1.2.

From (3.9) and (3.17), by applying the divergence theorem we get

$$
0=\int_{M} L(n H) \geq \int_{M}\left\{|\Phi|^{2}\left(n c-\frac{n}{2 \sqrt{n-1}} S\right)\right\} d M \geq 0 .
$$

Consequently, since we are supposing $S<2 \sqrt{n-1} c$, from (3.20) we obtain that $|\Phi|=$ 0 on $M^{n}$, and hence $M^{n}$ is totally umbilical.

Finally, taking into account the description of the totally umbilical spacelike hypersurfaces of $\mathbb{S}_{1}^{n+1}$ given by Montiel in Example 1 of [20], from Theorem 1.2 we get the following. 
COROLlARY 3.6. Let $M^{n}$ be a compact linear Weingarten spacelike hypersurface immersed in $\mathbb{S}_{1}^{n+1}$ such that $R=a H+b$ with $(n-1) a^{2}+4 n(1-b) \geq 0$. If $S<2 \sqrt{n-1}$, then $M^{n}$ is isometric to $\mathbb{S}^{n}$, up to scaling.

ACKNOWLEDGEMENTS. The first author is partially supported by CAPES/CNPq, Brazil, grant Casadinho/Procad 552.464/2011-2. The authors would like to thank the referee for giving some valuable suggestions which improved the paper.

\section{REFERENCES}

1. N. Abe, N. Koike and S. Yamaguchi, Congruence theorems for proper semi-Riemannian hypersurfaces in a real space form, Yokohama Math. J. 35 (1987), 123-136.

2. K. Akutagawa, On spacelike hypersurfaces with constant mean curvature in the de Sitter space, Math. Z. 196 (1987), 13-19.

3. H. Alencar and M. do Carmo, Hypersurfaces with constant mean curvature in spheres, Proc. Am. Math. Soc. 120 (1994), 1223-1229.

4. L. J. Alías, A. Brasil, Jr. and A. G. Colares, Integral formulae for spacelike hypersurfaces in conformally stationary spacetimes and applications, Proc. Edinburgh Math. Soc. 46 (2003), 465-488.

5. A. Brasil Jr., A. G. Colares and O. Palmas, A gap theorem for complete constant scalar curvature hypersurfaces in the de Sitter space, J. Geom. Phys. 37 (2001), 237250 .

6. A. Brasil Jr., A. G. Colares and O. Palmas, Complete spacelike hypersurfaces with constant mean curvature in the de Sitter space: A gap theorem, Illinois J. Math. 47 (2003), 847-866.

7. F. E. C. Camargo, R. M. B. Chaves and L. A. M. Sousa Jr., Rigidity theorems for complete spacelike hypersurfaces with constant scalar curvature in de Sitter space, Diff. Geom. Appl. 26 (2008), 592-599.

8. A. Caminha, A rigidity theorem for complete CMC hypersurfaces in Lorentz manifolds, Diff. Geom. Appl. 24 (2006), 652-659.

9. A. Caminha, The geometry of closed conformal vector fields on Riemannian spaces, Bull. Braz. Math. Soc. 42 (2011), 277-300.

10. É. Cartan, Familles de surfaces isoparamétriques dans les espaces à courbure constante, Ann. Mat. Pura Appl. 17 (1938), 177-191.

11. Q. M. Cheng and S. Ishikawa, Spacelike hypersurfaces with constant scalar curvature, Manuscripta Math. 95 (1998), 499-505.

12. S. Y. Cheng and S. T. Yau, Hypersurfaces with constant scalar curvature, Math. Ann. 225 (1977), 195-204.

13. S. M. Choi, S. M. Lyu and Y. J. Suh, Complete space-like hypersurfaces in a Lorentz manifold, Math. J. Toyama Univ. 22 (1999), 53-76.

14. A. J. Goddard, Some remarks on the existence of spacelike hypersurfaces of constant mean curvature, Math. Proc. Camb. Phil. Soc. 82 (1977), 489-495.

15. Z.-J. Hu, M. Scherfner and S.-J. Zhai, On spacelike hypersurfaces with constant scalar curvature in the de Sitter space, Diff. Geom. Appl. 25 (2007), 594-611.

16. H. Li, Global rigidity theorems of hypersurfaces, Ark. Mat. 35 (1997), 327-351.

17. H. Li, Y. J. Suh and G. Wei, Linear Weingarten hypersurfaces in a unit sphere, Bull. Korean Math. Soc. 46 (2009), 321-329.

18. A. Lichnerowicz, L'integration des équations de la gravitation relativiste et le problème n corps, J. Math. Pures Appl. 23 (1944), 37-63.

19. J. Liu and Z. Sun, On spacelike hypersurfaces with constant scalar curvature in locally symmetric Lorentz spaces, J. Math. Anal. Appl. 364 (2010), 195-203.

20. S. Montiel, An integral inequality for compact spacelike hypersurfaces in the de Sitter space and applications to the case of constant mean curvature, Indiana Univ. Math. J. 37 (1988), 909-917.

21. S. Montiel, A characterization of hyperbolic cylinders in the de Sitter space, Tôhoku Math. J. 48 (1996), 23-31. 
22. J. Ok Baek, Q. M. Cheng and Y. Jin Suh, Complete space-like hypersurfaces in locally symmetric Lorentz spaces, J. Geom. Phys. 49 (2004), 231-247.

23. M. Okumura, Hypersurfaces and a pinching problem on the second fundamental tensor, Am. J. Math. 96 (1974), 207-213.

24. Y. J. Suh, Y. S. Choi and H. Y. Yang, On space-like hypersurfaces with constant mean curvature in a Lorentz manifold, Houston J. Math. 28 (2002), 47-70.

25. S. T. Yau, Some function-theoretic properties of complete Riemannian manifolds and their applications to geometry, Indiana Univ. Math. J. 25 (1976), 659-670.

26. S. Zhang and B. Wu, Rigidity theorems for complete spacelike hypersurfaces with constant scalar curvature in locally symmetric Lorentz spaces, J. Geom. Phys. 60 (2010), 333340 . 